\title{
A discrete scheme for regularized anisotropic surface diffusion: a 6th order geometric evolution equation
}

\author{
FRANK HAUSSER ${ }^{\dagger}$ AND AXEL VOIGT ${ }^{\dagger}$ \\ Crystal Growth Group, Research Center Caesar, Ludwig-Erhard-Allee 2, 53175 Bonn, Germany
}

[Received 13 September 2004 and in revised form 1 June 2005]

\begin{abstract}
We study anisotropic surface diffusion of curves with a small corner energy regularization. The regularization allows the use of nonconvex free energy densities and turns the evolution law into a 6 th order geometric equation. Using a semi-implicit time discretization, we present a variational formulation of this equation for parametric curves, leading to a discretization based on linear finite elements. The resulting linear system is shown to be uniquely solvable. Numerical examples include the convergence of closed curves to the Wulff shape and the evolution of a thermodynamically unstable surface into a hill-valley structure and its subsequent coarsening.
\end{abstract}

2000 Mathematics Subject Classification: 35K55, 65M12, 65M15, 65M60.

Keywords: Surface diffusion; anisotropy; 6th order equations; parametric finite elements.

\section{Introduction}

Surface diffusion plays a crucial role in various fields of materials science. Applications include the spinodal decomposition and subsequent coarsening of a thermodynamically unstable crystal surface and the self-organization of nanostructures. If the crystal surface is driven by thermodynamics and is essentially independent of the bulk, surface diffusion is the dominant mass transport mechanism and determines the dynamics of the surface morphology. The evolution will tend to minimize the surface free energy and approach an equilibrium shape. In the following, we will consider a two-dimensional approximation of a crystal. The equilibrium shape ("Wulff shape") of a crystal in two dimensions is defined as the shape of minimum surface free energy $F=\int_{\Gamma} \gamma \mathrm{d} s$ under the constraint of fixed area [18]. The surface free energy density $\gamma=\gamma(\theta)$ depends on the local orientation $\theta$ of the surface normal, reflecting the anisotropy of the material. According to the Wulff theorem [27], the equilibrium shape may be constructed as follows: Draw at each point of the polar plot of $\gamma(\theta)$ a straight line perpendicular to the normal direction; the inner envelope of the resulting family of lines is geometrically similar to the equilibrium shape. Depending on the details of $\gamma$ the equilibrium shape may contain flat sides ("facets") and corners. Facets occur when $\gamma$ has cusps. Here we will concentrate on smooth free energy densities $\gamma$ leading to corners, which occurs if the stiffness $\tilde{\gamma}=\gamma+\gamma^{\prime \prime}$ becomes negative for some orientations. In this case it is energetically favourable to exclude high energy orientiations, i.e. the Wulff shape has missing orientations. As shown in [5], for positive stiffness, the Wulff shape may be parametrized as

$$
x(\theta)=\gamma^{\prime}(\theta) \cos (\theta)+\gamma(\theta) \sin (\theta), \quad y(\theta)=-\gamma^{\prime}(\theta) \sin (\theta)+\gamma(\theta) \cos (\theta) .
$$

\footnotetext{
†E-mail: hausser@caesar.de

‡E-mail: voigt@caesar.de
} 




FIG. 1. Equilibrium crystal shape ("Wulff shape") for a strong anisotropic surface energy $\gamma(\theta)=1.0+0.5 \cos (4 \theta)$; truncating the unphysical "ears" (dashed/blue) yields the equilibrium crystal shape (solid/red); the Wulff shape has sharp corners (missing orientiations) connected by almost flat sides. The constant chemical potential is chosen such that the area of the Wulff shape is $A=25 \pi$.

If the stiffness becomes negative for some orientations, the above parametrization has unphysical "ears"-see Fig. 11. In this case, the Wulff shape is obtained simply by removing these ears, as shown in [6]. While the equilibrium problem is well understood, modelling the dynamics can create severe difficulties in the case of strong anisotropy. In order to construct a dynamic model, the normal velocity $v$ of the surface is related to the chemical potential $\mu$, which is given as the variational derivative of the surface free energy $F$, namely $\mu=\delta F / \delta \Gamma=\tilde{\gamma} \kappa$, with $\tilde{\gamma}=\gamma+\gamma^{\prime \prime}$ being the surface stiffness, with the convention that the curvature $\kappa$ is positive for a convex curve, and the normal is pointing outward. Depending on the dominant mass transport mechanism two models are considered: For attachment kinetics one assumes that

$$
v=-\mu=-\tilde{\gamma} \kappa,
$$

i.e. the dynamics is described by anisotropic mean curvature flow. If surface diffusion is the dominant mass transport mechanism, the evolution law for $v$ is anisotropic surface diffusion

$$
v=\partial_{s}\left(\nu \partial_{s} \mu\right)=\partial_{s}\left(\nu \partial_{s}(\tilde{\gamma} \kappa)\right),
$$

with $v=v(\theta)$ being the mobility of atoms diffusing along the surface and $\partial_{s}$ denoting differentiation with respect to arclength $s$. If the surface stiffness $\tilde{\gamma}$ turns negative for certain angles both models become backward parabolic and therefore ill posed.

\subsection{Small corner energy regularization}

One way to overcome the resulting inherently unstable behaviour of the dynamic problem is to regularize the equation by adding a curvature dependent term to the surface free energy density. This has already been proposed on physical grounds in [17], and later mathematically introduced in [11]. In two dimensions the penalized interfacial energy density reads

$$
\gamma_{\epsilon}=\gamma+\frac{1}{2} \epsilon \kappa^{2}
$$

The basic idea for introducing a curvature dependent term is to penalize sharp corners. The corners get rounded on a small length scale given by $\epsilon^{1 / 2}$. Thus, minimizing the surface energy $F_{\epsilon}=$ $\int_{\Gamma} \gamma_{\epsilon} \mathrm{d} s$ will be a compromise between the two competing energy terms, which determines the rounding of the corner: a large curvature at the corner increases the regularization term, whereas a small curvature at the corner leads to orientations where $\gamma$ is large. The effect of the regularization 
on the equilibrium shape has been analysed in detail in [24]. In particular it is shown by asymptotic analysis that the equilibrium shapes for $\epsilon>0$ converge to the sharp corner $(\epsilon=0)$ equilibrium shape as $\epsilon \rightarrow 0$.

The regularized surface energy (4) leads to the chemical potential

$$
\mu_{\epsilon}=\frac{\delta F_{\epsilon}}{\delta \Gamma}=\tilde{\gamma} \kappa-\epsilon\left(\partial_{s s} \kappa+\frac{1}{2} \kappa^{3}\right) .
$$

Thus, in the case of attachment kinetics as the dominant mass transport mechanism one obtains the 4th order parabolic geometric evolution law

$$
v=-\mu_{\epsilon}=-\tilde{\gamma} \kappa+\epsilon\left(\partial_{S S} \kappa+\frac{1}{2} \kappa^{3}\right) .
$$

In the case of surface diffusion being the dominant mass transport mechanism the dynamics is described by the 6th order parabolic geometric evolution law

$$
v=\partial_{s}\left(v \partial_{s} \mu_{\epsilon}\right)=\partial_{s}\left(\nu \partial_{s}\left(\tilde{\gamma} \kappa-\epsilon\left(\partial_{s s} \kappa+\frac{1}{2} \kappa^{3}\right)\right)\right) .
$$

Note that if we set $\tilde{\gamma}=0$ and $\epsilon=1$, then (6) is known as Willmore flow. In this paper we will present a numerical scheme for (7) in a parametric formulation.

\subsection{Small slope approximation}

Before we describe our numerical approach, let us discuss a small slope approximation, which connects equations (6) and (7) to the well known Cahn-Hilliard equation. Here we closely follow [26], where the small slope approximation is derived for (6) with an additional driving force. Assume the planar front $\theta=0$ to be thermodynamically unstable, i.e. $\tilde{\gamma}(0)<0$. Moreover assume there exists a pair of stable orientations $\theta \sim \pm \eta$ with $0<\eta \ll 1$. In this case, a small slope approximation can be employed by expanding (6) and (7) in powers of $\eta$ as follows: Let $y=h(x, t)$ be the graph defining the surface; then $\partial_{x} h=\tan (\theta)$, where $-\pi / 2<\theta<\pi / 2$, is the surface slope. The projected energy is $V\left(\partial_{x} h\right):=\gamma(\theta) / \cos (\theta)$ and the second derivative of $V\left(\partial_{x} h\right)$ yields $V^{\prime \prime}\left(\partial_{x} h\right)=\cos ^{3}(\theta) \tilde{\gamma}(\theta)$. If we define the rescaled energy $W\left(\partial_{x} h / \eta\right):=V\left(\partial_{x} h\right)$ and expand $h=\eta h_{1}+\eta^{2} h_{2}+\cdots$ it follows that

$$
\begin{aligned}
v & =\eta \partial_{t} h_{1}+O\left(\eta^{2}\right), \\
\partial_{s} & =\partial_{x}+O\left(\eta^{2}\right), \\
\kappa & =-\eta \partial_{x x} h_{1}+O\left(\eta^{2}\right), \\
\partial_{s s} \kappa & =-\eta \partial_{x x x x} h_{1}+O\left(\eta^{2}\right), \\
\kappa^{3} & =O\left(\eta^{3}\right), \\
\tilde{\gamma}(\theta) & =W^{\prime \prime}\left(\partial_{x} h_{1}\right)+O(\eta) .
\end{aligned}
$$

Therefore we obtain for (6) and (7) in leading order

$$
\begin{aligned}
& \partial_{t} h_{1}=W^{\prime \prime}\left(\partial_{x} h_{1}\right) \partial_{x x} h_{1}-\epsilon \partial_{x x x x} h_{1}, \\
& \partial_{t} h_{1}=-\partial_{x}\left(v \partial_{x}\left(W^{\prime \prime}\left(\partial_{x} h_{1}\right) \partial_{x x} h_{1}-\epsilon \partial_{x x x x} h_{1}\right)\right)
\end{aligned}
$$

respectively. Differentiating (8) and (9) with respect to $x$ and setting $q=\partial_{x} h_{1}$ yields

$$
\begin{aligned}
& \partial_{t} q=\partial_{x x}\left(W^{\prime}(q)-\epsilon \partial_{x x} q\right), \\
& \partial_{t} q=-\partial_{x x}\left(v \partial_{x x}\left(W^{\prime}(q)-\epsilon \partial_{x x} q\right)\right)
\end{aligned}
$$


respectively. Equation (10) is identical to the Cahn-Hilliard equation describing spinodal decomposition in binary alloys and 111 is a higher order Cahn-Hilliard equation. The described long-wave approximations demonstrate the close connection between the regularized free energy $F_{\epsilon}=\int_{\Gamma}\left(\gamma(\theta)+\frac{1}{2} \epsilon \kappa^{2}\right) \mathrm{d} s$ and the free energy functional $E_{\epsilon}=\int_{[0, L]}\left(W(q)+\frac{1}{2} \epsilon\left|\partial_{x} q\right|^{2}\right) \mathrm{d} x$ from which the Cahn-Hilliard and the higher order Cahn-Hilliard equation can be derived. The small corner energy regularization $\frac{1}{2} \epsilon \kappa^{2}$ corresponds to the gradient term $\frac{1}{2} \epsilon\left|\partial_{x} q\right|^{2}$ and the analog to the surface free energy density $\gamma(\theta)$ is given by the rescaled projected energy $W(q)$.

To further demonstrate the connection we choose an example where a small slope approximation may be employed. Consider a surface energy density of the form

$$
\gamma(\theta)=1+a \cos (32 \theta), \quad \text { i.e. } \quad \tilde{\gamma}(\theta)=1+a\left(1-32^{2}\right) \cos (32 \theta) .
$$

Thus, choosing $a>\left(32^{2}-1\right)^{-1}$ leads to a Wulff shape with 32 corners. Fig. 2 presents the equilibrium shape and the projected energy $V$ for $a=10\left(32^{2}-1\right)^{-1}$. The corner orientations of the uppermost corner are determined by locating the smallest orientations $\theta^{ \pm}$, where the curve $(x(\theta), y(\theta))$ as given in Eq. (1) crosses itself, and are taken as the stable orientations $\pm \eta$. For $a=10\left(32^{2}-1\right)^{-1}$ one obtains $\theta^{ \pm}= \pm 0.089201$. In Fig. 3 (left) the equilibrium shape of the uppermost corner is depicted for different values of the anisotropy strength $a$, and Fig. 3 (right) shows the corresponding rescaled projected energies $W$ with $\eta=0.089201$. It clearly demonstrates the convex structure of $W$ if $\tilde{\gamma}(\theta)$ is nonnegative and the double-well structure of $W$ if the Wulff shape has missing orientations or equivalently if $\tilde{\gamma}(0)$ becomes negative. Note that even in this case, the Cahn-Hilliard equations can model only a small part of the phenomena of the full geometric evolution.


FIG. 2. Left: upper right quarter of the Wulff shape with area $A=25 \pi$ and anisotropy $\gamma=1+10\left(32^{2}-1\right)^{-1} \cos (32 \theta)$. Right: corresponding projected energy $V\left(\partial_{x} h\right)$.
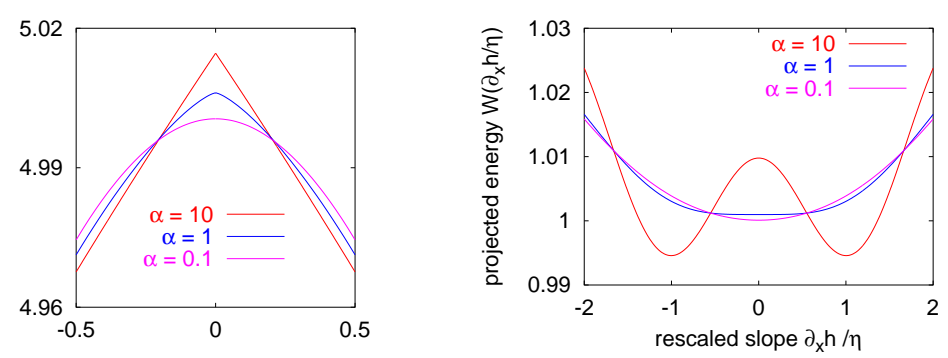

FIG. 3. Left: uppermost corner of the Wulf fshape with area $A=25 \pi$ and anisotropy $\gamma=1+\alpha\left(32^{2}-1\right)^{-1} \cos (32 \theta)$, with $\alpha=10, \alpha=1, \alpha=0.1$. Right: projected energies $W\left(\partial_{x} h / \eta\right)=V\left(\partial_{x} h\right)$, where $\eta=0.0892$ is the corner orientation for $\alpha=10$. 
Equations (10) and (11) are intensively studied to describe the dynamics of crystal surfaces [25, 19]. For both models thermodynamically unstable crystal surfaces undergo spinodal decomposition into a hill-valley pattern with stable orientations. These models attracted wide attention due to their role in self-organization of nanostructures. However, for such applications the long wave approximations are questionable because the small slope assumption on which they are based is not fulfilled. Therefore we will deal with the full nonlinear geometric evolution laws (6) and (7). The restriction to one-dimensional surfaces should be seen as a preliminary study to a full threedimensional theory. A finite element discretization for (6) has already been described in [16]. Here we will concentrate on a numerical solution of (77).

\section{$1.3 \quad$ Numerical approaches to surface diffusion}

Algorithms for surface diffusion are already discussed in the literature but are mainly restricted to the isotropic case. A level set method for isotropic surface diffusion was introduced in [8]. A semi-implicit discretization for the isotropic case is considered in [23] and for the anisotropic case in [9]. A graph formulation of (7) has been addressed recently in [4]. Starting with the analytic work of [7], which shows the formal convergence for vanishing interfacial thickness of a CahnHillard equation 11 with an increased mobility to the equation for motion by surface diffusion, several phase field approximations have been applied to study surface diffusion. The isotropic case is considered in [3, 20] and an anisotropic version is addressed in [13]. This approach also allows for a nonconvex surface free energy. Instead of the above described regularization here nonconvex angle intervals are simply excluded, which does not allow describing the formation of corners nor can it describe the nucleation of facets. A graph formulation for isotropic surface diffusion is addressed by finite elements in [1]. Weakly anisotropic surface diffusion in this context has been considered in [10]. In the parametric case finite element discretizations are introduced in [2] for the isotropic case and extended to the weakly anisotropic situation in [15]. Furthermore, several explicit finite difference schemes are applied to study surface diffusion, in the isotropic and anisotropic setting. A discretization of (7) in the parametric case has not been addressed with either method.

\section{Variational formulation and finite element discretization}

Following the ideas of [2], we start by writing the 6th order equation (7) as a system of 2nd order equations as follows: we introduce the position vector $\vec{x}$, the curvature vector $\vec{\kappa}=\kappa \vec{n}$, the weighted curvature $\tilde{\kappa}$, the variable $l$ and the velocity vector $\vec{v}=v \vec{n}$, and use the geometric expression $\vec{\kappa}=-\partial_{s s} \vec{x}$; then equation (7) becomes equivalent to the following system of equations for $\vec{\kappa}, \kappa, \tilde{\kappa}$, $l, v$, and $\vec{v}$ :

$$
\begin{aligned}
\vec{\kappa} & =-\partial_{s s} \vec{x}, \\
\kappa & =\vec{\kappa} \cdot \vec{n}, \\
\tilde{\kappa} & =\tilde{\gamma} \vec{\kappa} \cdot \vec{n}, \\
l & =\partial_{s s} \kappa+\frac{1}{2} \kappa^{3}, \\
v & =\partial_{s}\left(v \partial_{s}(\tilde{\kappa}-\epsilon l)\right), \\
\vec{v} & =v \vec{n} .
\end{aligned}
$$

\footnotetext{
${ }^{1}$ Instead of describing the slope of the phase boundary, as in Section 1.2 in this case the phase variable models the two bulk phases and the phase boundary is given by the zero level set.
} 
Let $\Gamma(t)$ denote the interface at time $t$. Now split the time interval by discrete time instants $0=t_{0}<t_{1}<\cdots$ and define time steps $\tau_{m}:=t_{m+1}-t_{m}$. We represent the next interface $\Gamma^{m+1}=\Gamma\left(t_{m+1}\right)$ in terms of $\Gamma^{m}=\Gamma\left(t_{m}\right)$ by updating the position vector

$$
\vec{x}^{m+1} \leftarrow \vec{x}^{m}+\tau_{m} \vec{v} .
$$

In the time discretization, all geometric quantities such as $\vec{n}$ and $\partial_{s}$ are evaluated on the current interface $\Gamma^{m}$. In contrast to the geometric quantities, the unknowns $\vec{\kappa}, \kappa, \tilde{\kappa}, l, v$, and $\vec{v}$ are treated implicitly, with the exception of the nonlinear term $\kappa^{3}$ which is treated semi-implicitly, i.e. $\kappa^{3}=\kappa^{m+1}\left(\kappa^{m}\right)^{2}$. In particular, in view of $(18)$, we define

$$
\vec{\kappa}^{m+1}=-\partial_{s s}\left(\vec{x}^{m}+\tau_{m} \vec{v}^{m+1}\right) .
$$

\subsection{Variational formulation}

To derive a weak formulation, we proceed similarly to [12, 2]: multiply $[13),(14),(15),(16),(17)$, and 19 by test functions $\vec{\psi} \in \vec{H}^{1}(\Gamma)$ and $\psi \in H^{1}(\Gamma)$, and use integration by parts. For simplicity, we have hereafter dropped the superscript $m+1$ for the unknowns $\vec{\kappa}^{m+1}$, etc. Furthermore, using the notation $\langle\cdot, \cdot\rangle$ for the $L^{2}$ inner product over the current interface $\Gamma^{m}$, we arrive at the following set of semi-implicit equations:

Problem 1 For $m=1,2, \ldots$ find $\vec{\kappa} \in \vec{H}^{1}\left(\Gamma^{m}\right), \kappa \in H^{1}\left(\Gamma^{m}\right), \tilde{\kappa} \in H^{1}\left(\Gamma^{m}\right), l \in H^{1}\left(\Gamma^{m}\right)$, $v \in H^{1}\left(\Gamma^{m}\right)$, and $\vec{v} \in \vec{H}^{1}\left(\Gamma^{m}\right)$ such that for all $\psi \in H^{1}\left(\Gamma^{m}\right)$ and all $\vec{\psi} \in \vec{H}^{1}\left(\Gamma^{m}\right)$,

$$
\begin{aligned}
\langle\vec{\kappa}, \vec{\psi}\rangle-\tau_{m}\left\langle\partial_{s} \vec{v}, \partial_{s} \vec{\psi}\right\rangle & =\left\langle\partial_{s} \vec{x}^{m}, \partial_{s} \vec{\psi}\right\rangle, \\
\langle\kappa, \psi\rangle-\langle\vec{\kappa} \cdot \vec{n}, \psi\rangle & =0, \\
\langle\tilde{\kappa}, \psi\rangle-\langle\tilde{\gamma} \vec{\kappa} \cdot \vec{n}, \psi\rangle & =0, \\
\langle l, \psi\rangle+\left\langle\partial_{s} \kappa, \partial_{s} \psi\right\rangle-\frac{1}{2}\left\langle\left(\kappa^{m}\right)^{2} \kappa, \psi\right\rangle & =0, \\
\langle v, \psi\rangle+\left\langle v \partial_{s} \tilde{\kappa}, \partial_{s} \psi\right\rangle-\epsilon\left\langle v \partial_{s} l, \partial_{s} \psi\right\rangle & =0, \\
\langle\vec{v}, \vec{\psi}\rangle-\langle v \vec{n}, \vec{\psi}\rangle & =0 .
\end{aligned}
$$

Now the discretization in space is straightforward: Consider a polygonal curve $\Gamma_{h}^{m}$ approximating $\Gamma^{m}$. The polygonal segments are thought of as finite elements. Also for the polygonal curve, we denote by $\vec{n}$ the outer unit normal to $\Gamma_{h}^{m}$, which may be discontinuous across inter-element boundaries. Denote by $\mathbb{W}_{h}^{m} \subseteq H^{1}\left(\Gamma_{h}^{m}\right)$ the finite element space of globally continuous, piecewise linear functions with corresponding nodal basis functions $\left(\psi_{l}\right)_{l=1}^{L}$, where $L$ is the number of degrees of freedom. By $\overrightarrow{\mathbb{W}}_{h}^{m} \subseteq \vec{H}^{1}\left(\Gamma_{h}^{m}\right)$ we denote the finite element space of vector-valued functions with nodal basis functions $\left(\vec{\psi}_{l}^{q}\right)_{l=1, \ldots, L}^{q=1,2}$, where $\vec{\psi}_{l}^{q}=\psi_{l} \vec{e}_{q}$ with $\psi_{l}$ the scalar basis function defined above and $\left(\vec{e}_{1}, \vec{e}_{2}\right)$ the standard basis in $\mathbb{R}^{2}$. Problem 1 is discretized by expanding the functions $\vec{\kappa}$, $\kappa, \tilde{\kappa}, l, v, \vec{v}$ in terms of the basis functions and testing against all discrete test functions, i.e. solving Problem 1 in the finite-dimensional spaces $\mathbb{W}_{h}^{m}, \overrightarrow{\mathbb{W}}_{h}^{m}$.

To arrive at an algorithm in a block matrix form, we introduce an auxiliary variable $i$ being identically 0 , i.e. $i=0$, and expand the unknowns

$$
\vec{\kappa}_{h}=\sum_{l=1}^{L} \vec{K}_{l} \psi_{l}, \quad \kappa_{h}=\sum_{l=1}^{L} K_{l} \psi_{l},
$$




$$
\begin{aligned}
\tilde{\kappa}_{h} & =\sum_{l=1}^{L} \tilde{K}_{l} \psi_{l}, \quad l_{h}=\sum_{l=1}^{L} L_{l} \psi_{l}, \\
\vec{v}_{h} & =\sum_{l=1}^{L} \vec{V}_{l} \psi_{l}, \quad v_{h}=\sum_{l=1}^{L} V_{l} \psi_{l}, \\
i_{h} & =\sum_{l=1}^{L} I_{l} \psi_{l}
\end{aligned}
$$

for some

$$
\begin{array}{ll}
\vec{K}=\left(\vec{K}_{1}, \ldots, \vec{K}_{L}\right)^{t} \in \mathbb{R}^{2 \times L}, & K=\left(K_{1}, \ldots, K_{L}\right)^{t} \in \mathbb{R}^{L}, \\
\tilde{K}=\left(\tilde{K}_{1}, \ldots, \tilde{K}_{L}\right)^{t} \in \mathbb{R}^{L}, & L=\left(L_{1}, \ldots, L_{L}\right)^{t} \in \mathbb{R}^{L}, \\
\vec{V}=\left(\vec{V}_{1}, \ldots, \vec{V}_{L}\right)^{t} \in \mathbb{R}^{2 \times L}, & V=\left(V_{1}, \ldots, V_{L}\right)^{t} \in \mathbb{R}^{L}, \\
I=\left(I_{1}, \ldots, I_{L}\right)^{t} \in \mathbb{R}^{L}, &
\end{array}
$$

and define the mass, stiffness, and normal matrices:

$$
\begin{array}{ll}
\boldsymbol{M}=\left(M_{k l}\right), & M_{k l}=\left\langle\psi_{k}, \psi_{l}\right\rangle, \\
\overrightarrow{\boldsymbol{M}}=\left(\vec{M}_{k l}\right), & \vec{M}_{k l}=\left(M_{k l}^{q r}\right)=\left(\delta_{q r} M_{k l}\right), \\
\boldsymbol{M}_{\kappa}=\left(M_{\kappa, k l}\right), & M_{\kappa, k l}=\left\langle\left(\kappa^{m}\right)^{2} \psi_{k}, \psi_{l}\right\rangle, \\
\boldsymbol{A}=\left(A_{k l}\right), & A_{k l}=\left\langle\partial_{s} \psi_{k}, \partial_{s} \psi_{l}\right\rangle, \\
\overrightarrow{\boldsymbol{A}}=\left(\vec{A}_{k l}\right), & \vec{A}_{k l}=\left(A_{k l}^{q r}\right)=\left(\delta_{q r} A_{k l}\right), \\
\boldsymbol{A}_{v}=\left(A_{v, k l}\right), & A_{v, k l}=\left\langle v \partial_{s} \psi_{k}, \partial_{s} \psi_{l}\right\rangle, \\
\overrightarrow{\boldsymbol{N}}=\left(\vec{N}_{k l}\right), & \vec{N}_{k l}=\left(N_{k l}^{q}\right)=\left\langle\psi_{k}, \psi_{l} n^{q}\right\rangle, \\
\overrightarrow{\boldsymbol{N}}_{\tilde{\gamma}}=\left(\vec{N}_{\tilde{\gamma}, k l}\right), & \vec{N}_{\tilde{\gamma}, k l}=\left(N_{\tilde{\gamma}, k l}^{q}\right)=\left\langle\psi_{k}, \tilde{\gamma} \psi_{l} n^{q}\right\rangle,
\end{array}
$$

where the index ranges are $1 \leqslant k, l \leqslant L$ and $1 \leqslant q, r \leqslant 2, \delta_{q r}=\vec{e}_{q} \cdot \vec{e}_{r}$ is the Kronecker symbol, and $n^{q}=\vec{n} \cdot \vec{e}_{q}$ is the $q$-th spatial component of the normal.

The matrix form of the discretized Problem 1 reads: Find $\vec{K}, \vec{V} \in \mathbb{R}^{2 \times L}, K, \tilde{K}, L, I, V \in \mathbb{R}^{L}$ such that $\Pi \boldsymbol{\lambda}=\boldsymbol{\eta}$ with

$$
\begin{aligned}
\boldsymbol{\lambda} & =(\vec{V}, K, L, I, \tilde{K}, \vec{K}, V)^{t}, \quad \eta=(0,0,0,0,0,0, \vec{A} \vec{X}, 0)^{t}, \\
\boldsymbol{\Pi} & =\left(\begin{array}{ccc}
\boldsymbol{\Gamma} & 0 & \boldsymbol{\Delta} \\
\boldsymbol{\Theta} & \boldsymbol{\Gamma} & \boldsymbol{\Lambda} \\
\Xi & \boldsymbol{\Sigma} & \boldsymbol{\Gamma}
\end{array}\right)
\end{aligned}
$$

with

$$
\begin{aligned}
& \Gamma=\left(\begin{array}{ccc}
M & 0 & 0 \\
0 & M & 0 \\
0 & 0 & M
\end{array}\right), \\
& \Delta=\left(\begin{array}{ccc}
0 & 0 & -N^{1} \\
0 & 0 & -N^{2} \\
-N^{1} & -N^{2} & 0
\end{array}\right), \\
& \Theta=\left(\begin{array}{lll}
0 & 0 & A-\frac{1}{2} M_{\kappa} \\
0 & 0 & 0 \\
0 & 0 & 0
\end{array}\right), \\
& \boldsymbol{\Lambda}=\left(\begin{array}{ccc}
\mathbf{0} & \mathbf{0} & \mathbf{0} \\
\mathbf{0} & \mathbf{0} & \mathbf{0} \\
-\boldsymbol{N}_{\tilde{\gamma}}^{1} & -\boldsymbol{N}_{\tilde{\gamma}}^{2} & \mathbf{0}
\end{array}\right), \\
& \Xi=\left(\begin{array}{ccc}
-\tau_{m} \boldsymbol{A} & \mathbf{0} & \mathbf{0} \\
\mathbf{0} & -\tau_{m} \boldsymbol{A} & \mathbf{0} \\
\mathbf{0} & \mathbf{0} & \mathbf{0}
\end{array}\right) \text {, } \\
& \Sigma=\left(\begin{array}{ccc}
0 & 0 & 0 \\
0 & 0 & 0 \\
-\epsilon A_{\nu} & 0 & A_{\nu}
\end{array}\right) \text {. }
\end{aligned}
$$


A simple Gauß elimination now leads to

$$
\left(\boldsymbol{\Gamma}-\boldsymbol{\Xi} \boldsymbol{\Gamma}^{-1} \boldsymbol{\Delta}-\boldsymbol{\Sigma} \boldsymbol{\Gamma}^{-1} \boldsymbol{\Lambda}+\boldsymbol{\Sigma} \boldsymbol{\Gamma}^{-1} \boldsymbol{\Theta} \boldsymbol{\Gamma}^{-1} \boldsymbol{\Delta}\right)\left(\begin{array}{c}
\overrightarrow{\boldsymbol{K}} \\
V
\end{array}\right)=\left(\begin{array}{c}
\overrightarrow{\boldsymbol{A}} \overrightarrow{\boldsymbol{X}} \\
0
\end{array}\right),
$$

which is equivalent to

$$
\left(\begin{array}{cc}
\vec{M} & -\tau_{m} \vec{A} M^{-1} \vec{N} \\
A_{v} M^{-1} \overrightarrow{\boldsymbol{N}}_{\tilde{\gamma}}^{t}+\epsilon \boldsymbol{A}_{v} \boldsymbol{M}^{-1}\left(\boldsymbol{A}-\frac{1}{2} \boldsymbol{M}_{\kappa}\right) M^{-1} \overrightarrow{\boldsymbol{N}}^{t} & \boldsymbol{M}
\end{array}\right)\left(\begin{array}{c}
\overrightarrow{\boldsymbol{K}} \\
V
\end{array}\right)=\left(\begin{array}{c}
\overrightarrow{\boldsymbol{A}} \overrightarrow{\boldsymbol{X}} \\
0
\end{array}\right)
$$

and gives rise to the final Schur complement equation for $V$,

$$
\begin{aligned}
\left(\tau_{m}\left(\boldsymbol{A}_{v} \boldsymbol{M}^{-1} \overrightarrow{\boldsymbol{N}}_{\tilde{\gamma}}^{t}+\epsilon \boldsymbol{A}_{\nu} \boldsymbol{M}^{-1}\left(\boldsymbol{A}-\frac{1}{2} \boldsymbol{M}_{\kappa}\right) \boldsymbol{M}^{-1} \overrightarrow{\boldsymbol{N}}^{t}\right) \overrightarrow{\boldsymbol{M}}^{-1} \overrightarrow{\boldsymbol{A}} \boldsymbol{M}^{-1} \overrightarrow{\boldsymbol{N}}+\boldsymbol{M}\right) V \\
=-\left(\boldsymbol{A}_{v} \boldsymbol{M}^{-1} \overrightarrow{\boldsymbol{N}}_{\tilde{\gamma}}^{t}+\epsilon \boldsymbol{A}_{v} \boldsymbol{M}^{-1}\left(\boldsymbol{A}-\frac{1}{2} \boldsymbol{M}_{\kappa}\right) \boldsymbol{M}^{-1} \overrightarrow{\boldsymbol{N}}^{t}\right) \vec{M}^{-1} \overrightarrow{\boldsymbol{A}} \vec{X}^{m} .
\end{aligned}
$$

Once the scalar velocity $V$ is obtained by solving [20, the unknown $\vec{V}$ is easily computed by solving $\vec{M} \vec{V}=\vec{N} V$, and then $\vec{X}$ is updated through

$$
\vec{X} \leftarrow \vec{X}+\tau_{m} \vec{V}
$$

\subsection{Solvability of the linear system}

In order to show uniqueness of the discrete system it is sufficient to show that if $\left(\vec{\kappa}_{h}, \kappa_{h}, \tilde{\kappa}_{h}, l_{h}, \vec{v}_{h}, v_{h}\right)$ satisfies

$$
\begin{aligned}
\left\langle\vec{\kappa}_{h}, \vec{\psi}_{h}\right\rangle-\tau_{m}\left\langle\partial_{s} \vec{v}_{h}, \partial_{s} \vec{\psi}_{h}\right\rangle & =0, \\
\left\langle\kappa_{h}, \psi_{h}\right\rangle-\left\langle\vec{\kappa}_{h} \cdot \vec{n}, \psi_{h}\right\rangle & =0, \\
\left\langle\tilde{\kappa}_{h}, \psi_{h}\right\rangle-\left\langle\tilde{\gamma} \vec{\kappa}_{h} \cdot \vec{n}, \psi_{h}\right\rangle & =0, \\
\left\langle l_{h}, \psi_{h}\right\rangle+\left\langle\partial_{s} \kappa_{h}, \partial_{s} \psi_{h}\right\rangle-\frac{1}{2}\left\langle\left(\kappa_{h}^{m}\right)^{2} \kappa_{h}, \psi_{h}\right\rangle & =0, \\
\left\langle v_{h}, \psi_{h}\right\rangle+\left\langle v \partial_{s} \tilde{\kappa}_{h}, \partial_{s} \psi_{h}\right\rangle-\epsilon\left\langle v \partial_{s} l_{h}, \partial_{s} \psi_{h}\right\rangle & =0, \\
\left\langle\vec{v}_{h}, \vec{\psi}_{h}\right\rangle-\left\langle v_{h} \vec{n}, \vec{\psi}_{h}\right\rangle & =0,
\end{aligned}
$$

then $\left(\vec{\kappa}_{h}, \kappa_{h}, \tilde{\kappa}_{h}, l_{h}, \vec{v}_{h}, v_{h}\right)=0$. This is shown by subsequently testing the above identities with the unknowns and using the inverse inequality

$$
\left\|\partial_{s} f_{h}\right\|_{L^{2}\left(\Gamma_{h}^{m}\right)} \leqslant C h^{-1}\left\|f_{h}\right\|_{L^{2}\left(\Gamma_{h}^{m}\right)},
$$

where $h$ is a lower bound on the lengths of the segments of $\Gamma_{h}^{m}$ and $C$ is a generic constant. In the following $\|\cdot\|$ denotes the $L^{2}\left(\Gamma_{h}^{m}\right)$ norm. First insert $\vec{\psi}_{h}=\vec{\kappa}_{h}$ into 21) to get

$$
\left\|\vec{\kappa}_{h}\right\|^{2} \leqslant \tau_{m}\left\|\partial_{s} \vec{v}_{h}\right\|\left\|\partial_{s} \vec{\kappa}_{h}\right\| \leqslant C \tau_{m} h^{-2}\left\|\vec{v}_{h}\right\|\left\|\vec{\kappa}_{h}\right\| .
$$

Next, choosing $\vec{\psi}_{h}=\vec{v}_{h}$ in 26) yields $\left\|\vec{v}_{h}\right\| \leqslant\left\|v_{h}\right\|$. Plugging this identity into 28) leads to

$$
\left\|\vec{\kappa}_{h}\right\| \leqslant C \tau_{m} h^{-2}\left\|v_{h}\right\| .
$$


Now we choose $\psi_{h}=v_{h}$ in 25 to get

$$
\left\|v_{h}\right\|^{2} \leqslant C\left\|\partial_{s} \tilde{\kappa}_{h}\right\|\left\|\partial_{s} v_{h}\right\|+C \epsilon\left\|\partial_{s} l_{h}\right\|\left\|\partial_{s} v_{h}\right\| \leqslant C h^{-2}\left\|\tilde{\kappa}_{h}\right\|\left\|v_{h}\right\|+C \epsilon h^{-2}\left\|l_{h}\right\|\left\|v_{h}\right\|,
$$

where we assume $|v| \leqslant C$. Dividing by $\left\|v_{h}\right\|$ yields

$$
\left\|v_{h}\right\| \leqslant C h^{-2}\left\|\tilde{\kappa}_{h}\right\|+C h^{-2} \epsilon\left\|l_{h}\right\| .
$$

Choosing $\psi_{h}=l_{h}$ in 24) gives

$$
\begin{aligned}
\left\|l_{h}\right\|^{2} & \leqslant\left\|\partial_{s} \kappa_{h}\right\|\left\|\partial_{s} l_{h}\right\|+C\left\|\kappa_{h}\right\|\left\|l_{h}\right\| \leqslant C h^{-2}\left\|\kappa_{h}\right\|\left\|l_{h}\right\|+C\left\|\kappa_{h}\right\|\left\|l_{h}\right\| \\
& \leqslant C\left(1+h^{-2}\right)\left\|\kappa_{h}\right\|\left\|l_{h}\right\|,
\end{aligned}
$$

where we assume $\left(\kappa_{h}^{m}\right)^{2} \leqslant C$. This implies

$$
\left\|l_{h}\right\| \leqslant C\left(1+h^{-2}\right)\left\|\kappa_{h}\right\| .
$$

Finally, setting $\psi_{h}=\kappa_{h}$ in 22) yields $\left\|\kappa_{h}\right\| \leqslant\left\|\vec{\kappa}_{h}\right\|$ and choosing $\psi_{h}=\tilde{\kappa}_{h}$ in 23) implies $\left\|\tilde{\kappa}_{h}\right\| \leqslant C\left\|\vec{\kappa}_{h}\right\|$, where we assume $|\tilde{\gamma}|<C$. These inequalities together with 30] and (31) imply

$$
\left\|v_{h}\right\| \leqslant C h^{-2}\left(1+\epsilon\left(1+h^{-2}\right)\right)\left\|\vec{\kappa}_{h}\right\| .
$$

Inserting the last inequality into (29) finally leads to $\vec{\kappa}_{h}=0$, provided that

$$
C \tau_{m} h^{-4}\left(1+\epsilon\left(1+h^{-2}\right)\right)<1,
$$

with $C$ depending on $\nu, \kappa_{h}^{m}$ and $\tilde{\gamma}$. Now, equations $30,31,32$ may be used to show that also the other unknowns are identically zero.

The time step restriction (33) indicates that the parameters $\epsilon, \tau_{m}$ and $h$ may not be chosen independently of each other and the way in which they are related will certainly influence the stability of the scheme. In our model $\epsilon$ is a small parameter and $\sqrt{\epsilon}$ is setting the length scale of the rounded corners (see Section 3 for a numerical verification). In order to resolve this length scale we therefore need $h^{2} \sim \epsilon$. This means that in practice we have a time step restriction $\tau_{m} \sim h^{4}$, instead of $h^{6}$ for an explicit scheme. Such a restriction on the time step is also observed in the numerical results (see Section 3 ).

\section{Numerical results}

In this section we present numerical results for the anisotropic regularized surface diffusion as defined in (7). The numerical method is implemented using ALBERT, an adaptive finite element software for scientific computation [22]. We use an area preserving local mesh regularization as described in [2] to keep the mesh size approximately constant during the simulation. If not stated otherwise, we use the nonconvex anisotropy function

$$
\gamma(\theta)=1.0+0.5 \cos (4 \theta), \quad \text { i.e. } \quad \tilde{\gamma}(\theta)=1.0-7.5 \cos (4 \theta),
$$

which exibits a four-fold symmetry (see Fig. 11. We investigate the convergence of a circle to the Wulff shape and also the influence of the value of the regularization parameter $\epsilon$. Furthermore, the evolution of a thermodynamically unstable crystal surface into a hill-valley structure and its subsequent coarsening are analysed. 


\subsection{Convergence to the equilibrium shape}

Since the chemical potential is constant along the equilibrium shape, the equilibrium shape is a stationary solution of (7). Therefore it is assumed that a closed curve which is close to the equilibrium shape will converge to this shape under (regularized) anisotropic surface diffusion. To verify this assumption, the evolution of a circle of radius $r=5$ is simulated (see Fig. 4). The parameter $\epsilon$ takes the values $1.0,0.25$ and 0.1 . For $\epsilon=1.0,0.25$, the number of grid points is $n=256$ and the time step is $\tau=10^{-5}$. For $\epsilon=0.1, n=512$ and the time step is chosen adaptively depending on the maximal normal velocity, where the maximal time step is $\tau=10^{-5}$. In all three cases, the closed curve converges to a stationary shape. Note that for $\epsilon=0.1$ a hill-valley structure emerges at the early stage at the four unstable orientations $\theta=0, \pi / 2, \pi, 3 \pi / 2$. The resulting hillvalley patterns coarsen, which finally leads to a stationary shape. This behaviour will be analysed in more detail in Section 3.2
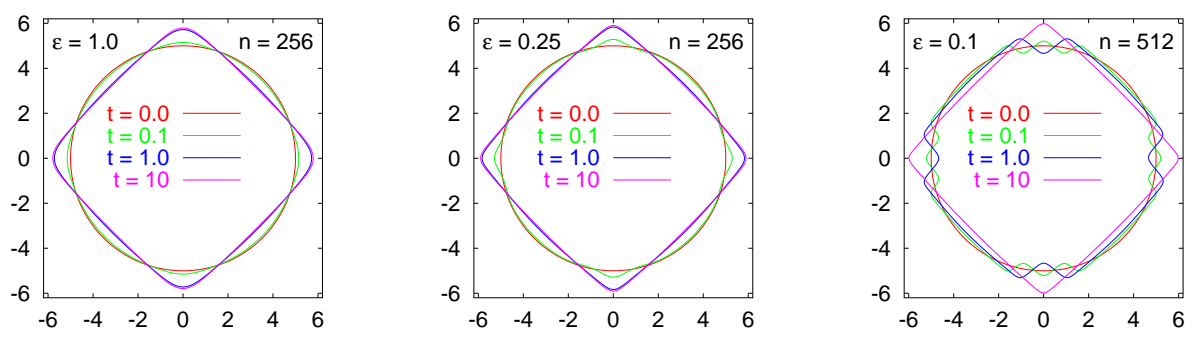

FIG. 4. Time evolution of a closed curve for different parameters $\epsilon$. Initial curve is a circle with radius $r=5$.

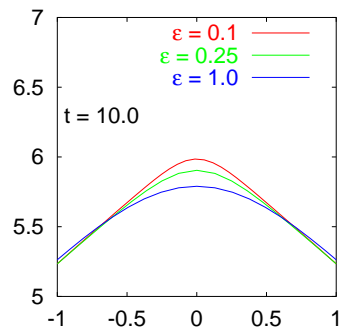

FIG. 5. Upper corner of the solutions at $t=10$ for different values of $\epsilon$.

For the stationary shape, we expect the length scale of the rounded corner to be $\sim \epsilon^{1 / 2}$, which is in agreement with the numerical results (see Fig. 5). A fundamental property of motion by surface diffusion is conservation of area of the enclosed region, which is also satisfied by the regularized anisotropic surface diffusion, since

$$
\frac{\mathrm{d}}{\mathrm{d} t}|\Omega(t)|=\int_{\Gamma(t)} v=\int_{\Gamma(t)} \partial_{s}\left(v \partial_{s} \mu_{\epsilon}\right)=\int_{\Gamma(t)} v \partial_{s} \mu_{\epsilon} \partial_{s} 1=0 .
$$

Also, starting from a circular shape, the curve length is expected to increase while approaching the stationary shape. Figure 6 shows the enclosed area and the length of the curve over time. The simulation results clearly show the approach of a stationary solution. The area is conserved within 

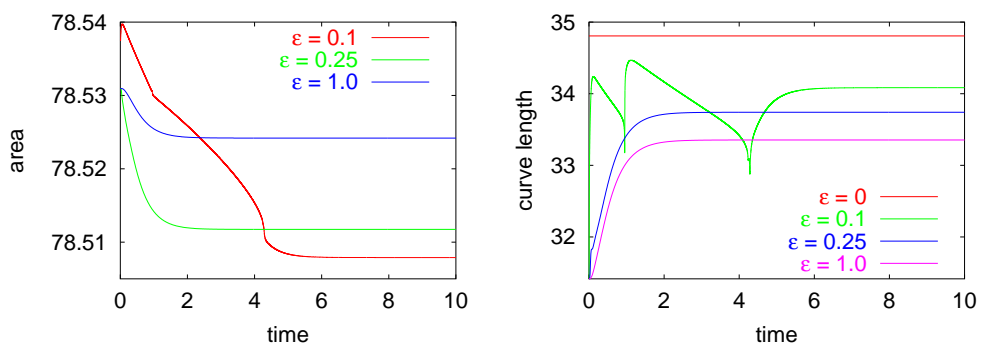

FIG. 6. Simulations of equation 7 with different values of $\epsilon$ : (left) area conservation, (right) curve length over time and curve length of the Wulff shape for $\epsilon=0$.

$0.05 \%$ and the length of the curve approaches the length of the equilibrium shape with sharp corners (i.e. $\epsilon=0$ ) as $\epsilon$ decreases.

In order to analyse the resulting stationary shapes in more detail, we compare the numerical solutions with the asymptotic solutions for the equilibrium shapes as given in [24]. Here the idea is to take the sharp corner equilibrium shape $(\epsilon=0)$ as the outer solution and to derive an inner solution for the equlibrium shape near the corner as an expansion in $\epsilon^{1 / 2}$, which rounds the corner. Let us briefly review the derivation of the inner solution as given in [24]: First recall that an equilibrium shape is a solution of (5) with constant chemical potential $\mu_{\epsilon}$. Now let $s$ be the arclength with $s=0$ at the corner of the outer solution and $\Theta(S):=\theta\left(s / \epsilon^{1 / 2}\right)$ the rescaled local orientation. Expanding $\Theta(S)=\Theta_{0}(S)+\epsilon^{1 / 2} \Theta_{1}(S)+\cdots$ and using the identity $\kappa=\mathrm{d} \theta / \mathrm{d} s$ one obtains from (5) in leading order

$$
\frac{\mathrm{d}^{3} \Theta_{0}}{\mathrm{~d} S^{3}}-\tilde{\gamma}\left(\Theta_{0}\right) \frac{\mathrm{d} \Theta_{0}}{\mathrm{~d} S}+\frac{1}{2}\left(\frac{\mathrm{d} \Theta_{0}}{\mathrm{~d} S}\right)^{3}=0 .
$$

Moreover, to match the outer solution, the following boundary conditions have to be fulfilled:

$$
\Theta_{0} \rightarrow \theta_{c}^{ \pm} \quad \text { as } S \rightarrow \pm \infty
$$

where $\theta_{c}^{ \pm}$are the corner orientations of the outer solution. Passing to the new variable $Q\left(\Theta_{0}\right):=$ $\frac{1}{2}\left(\mathrm{~d} \Theta_{0} / \mathrm{d} S\right)^{2}$, one obtains from 35 the linear problem

$$
Q+\frac{\mathrm{d}^{2} Q}{\mathrm{~d} \Theta^{2}}=\tilde{\gamma}\left(\Theta_{0}\right)
$$

with boundary conditions

$$
Q\left(\theta_{c}^{ \pm}\right)=0, \quad \frac{\mathrm{d} Q}{\mathrm{~d} \Theta_{0}}\left(\theta_{c}^{ \pm}\right)=0 .
$$

Thus, recalling that $\tilde{\gamma}=\gamma+\gamma^{\prime \prime}$, a particular solution is just $Q=\gamma$ and the general solution is

$$
Q\left(\Theta_{0}\right)=\gamma\left(\Theta_{0}\right)+A \cos \left(\Theta_{0}\right)+B \sin \left(\Theta_{0}\right) .
$$

Sticking to the symmetric case, i.e. $\theta_{c}:=\theta_{c}^{+}=-\theta_{c}^{-}$, one obtains $B=0$ and

$$
A=-\gamma\left(\theta_{c}\right) \cos \left(\theta_{c}\right)+\gamma^{\prime}\left(\theta_{c}\right) \sin \left(\theta_{c}\right) .
$$


Finally, $S\left(\Theta_{0}\right)$ is obtained by integration as

$$
S\left(\Theta_{0}\right)=\int_{0}^{\Theta_{0}} \frac{1}{\sqrt{2 Q(\phi)}} \mathrm{d} \phi .
$$

Inverting $S\left(\Theta_{0}\right)$ yields $\Theta_{0}(S)$. As shown in [24], if the outer solution has a corner, the inner solution exists and is uniquely determined by the corner orientations $\theta_{c}^{ \pm}$. The composite solution in the neighborhood of a corner with inner solution $\theta_{\text {inner }}$ is then given by

$$
\theta(s)=\theta_{\text {outer }}(s)+\theta_{\text {inner }}(s)-\theta_{\text {match }},
$$

where (assuming $s=0$ at the corner) $\theta_{\text {match }}=\theta_{c}^{+}$(resp. $\theta_{\text {match }}=\theta_{c}^{-}$) for $s>0$ (resp. $s<0$ ).

In Fig. 7, the outer and the composite solution for the equilibrium shape with surface energy given in (34) is depicted for the uppermost corner. The outer solution, i.e., the sharp corner solution, has a discontinuity at the corner and the composite solution smoothes the jump over a transition layer of thickness $O\left(\epsilon^{1 / 2}\right)$. One should note that the shape defined by the composite solution has a slightly smaller area then the sharp corner equlibrium shape.

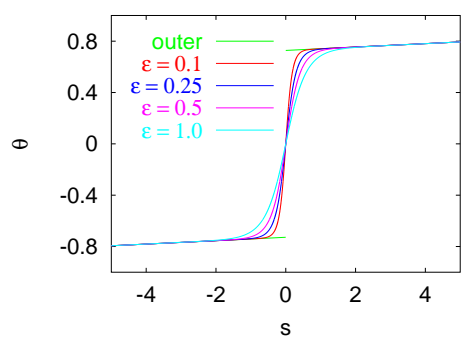

FIG. 7. Asymptotic solution of [24] for corner with regularization. Shown is the orientation $\theta$ versus arclength $s$ for the anisotropic surface energy given in 34. The outer solution is the equilibrium solution with $\epsilon=0$ and the composite solution is depicted for different values of $\epsilon$. The area of the sharp corner equilibrium shape is chosen to be $A=25 \pi$.
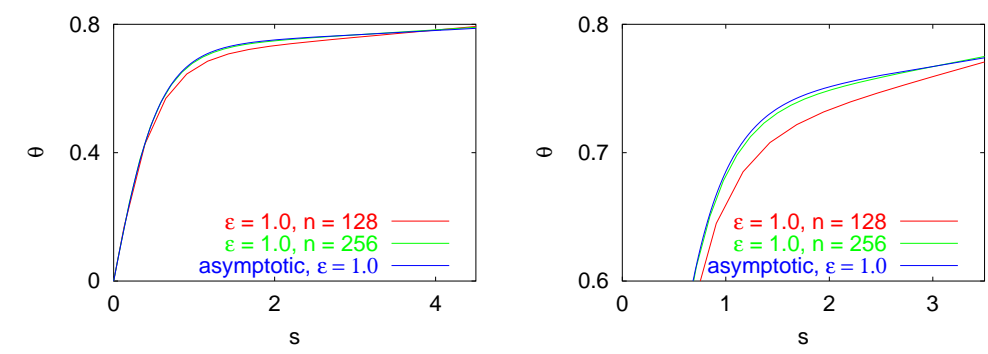

FIG. 8. Approaching the asymptotic solution. Left: $\theta(s)$ of the stationary shape (numerical solution of (7) at $t=10)$ at a rounded corner for different numbers of grid points $n$. Simulations for $\epsilon=1.0$. Right: zooming in.

We now compare our stationary solution (numerical solution of $(7)$ at $t=10$ ) with these asymptotic solutions. As presented in Figs. 8 and 9, the numerical solution approaches the asymptotic solution for decreasing grid size $h$. 

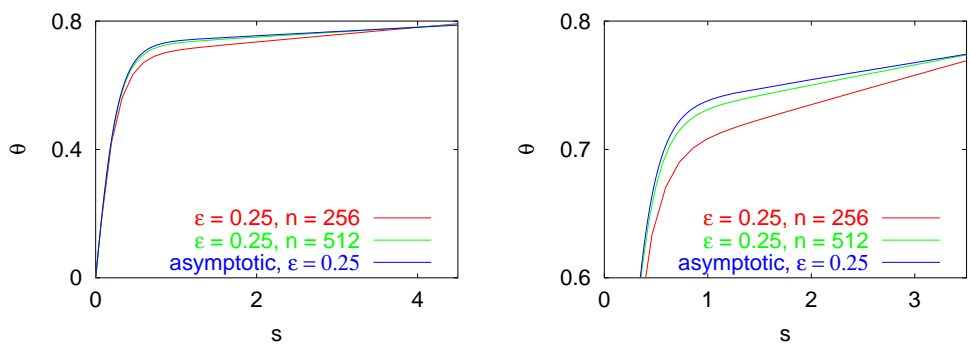

FIG. 9. Approaching the asymptotic solution. Left: $\theta(s)$ of the stationary shape (numerical solution of (7) at $t=10)$ at a rounded corner for different numbers of grid points $n$. Simulations for $\epsilon=0.25$. Right: zooming in.

To investigate how the regularization affects the equilibrium shape if the stiffness $\tilde{\gamma}$ is strictly positive, we have performed simulations with a convex surface energy $\gamma=1+0.03 * \cos (4 \theta)$ and for different values of $\epsilon$. Starting from a circle of radius $r=5$, the curves evolve to a stationary shape. Plots of the local angle versus arclength of the solutions at $t=10$ for $0 \leqslant \theta \leqslant \pi / 4$ are depicted in Fig. 10 Comparing with the equilibrium solution for $\epsilon=0$, it can be seen that the curvature dependent higher order term in equation (7) has a very small effect on the equilibrium shape if $\epsilon$ is small, as long as there are no corners in the Wulff shape, which justifies neglecting higher order terms if weak anisotropies are considered.



FIG. 10. Influence of regularization in the case of positive stiffness $(\tilde{\gamma}>0): \theta(s)$ of the stationary solution (numerical solution of (7) at $t=10$ ) for $0 \leqslant \theta \leqslant \pi / 4$. The initial curve is a circle with radius $r=5$. Surface energy is $\gamma=$ $1+0.03 * \cos (4 \theta)$

\subsection{Spinodal decomposition of a thermodynamically unstable surface}

As a second example we analyse the unstable behaviour observed in Figure 4 for $\epsilon=0.1$ in more detail. The observed structure for $t=0.1$ and 1.0 results from the unstable orientations $\theta=0, \pi / 2, \pi$ and $3 \pi / 2$. We concentrate on the unstable orientation $\theta=0$ and perform simulations on an interval $[0, L]$ with periodic boundary conditions. Fig. 11 shows the evolution of a randomly perturbed plane surface, which undergoes spinodal decomposition into a hill-valley structure. At the first stage a hill-valley structure with rapidly growing amplitudes emerges. At a second stage, the evolution of the surface is governed by the nonlinear interaction of the hills and valleys. The average distance to neighbouring hill increases due to the annihilation of hills and valleys. This coarsening dynamics is similar to the one-dimensional coarsening observed in the higher order Cahn-Hilliard equation (11) [19, 21]. 


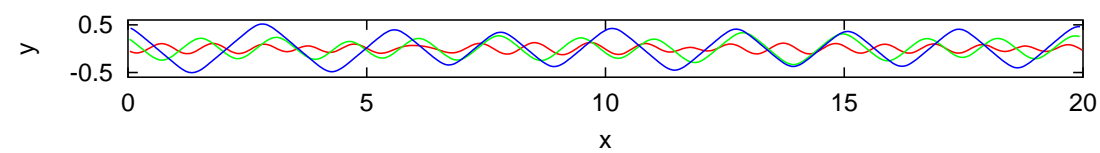

FIG. 11. Spinodal decomposition and coarsening: time evolution of a randomly perturbed straight line on a periodic domain of length $L=20$. The solution is shown at $t=0.005,0.5,50$.

Let us analyse the emerging of the hill-valley pattern in more detail. Setting $v=1$ and linearizing (9) around the unstable plane solution $\theta=0$ one obtains

$$
\partial_{t} h=\partial_{x x}\left(W^{\prime \prime}(0) \partial_{x x} h-\epsilon \partial_{x x x x} h\right) .
$$

This equation has plane wave solutions $h_{k}(x, t)$ given by

$$
h_{k}=c \exp (\omega(k) t) \cos (k x), \quad \omega(k)=-W^{\prime \prime}(0) k^{4}-\epsilon k^{6} .
$$

Unstable modes $k$ obeying $\omega(k)>0$ are given by $k<\sqrt{\left|W^{\prime \prime}(0)\right| / \epsilon}$. The maximal growth rate is obtained for

$$
k_{\max }=\sqrt{2\left|W^{\prime \prime}(0)\right| / 3 \epsilon} \quad \text { yielding } \quad \omega_{\max }=4\left|W^{\prime \prime}(0)\right|^{3} / 27 \epsilon^{2} .
$$

Thus, the most unstable wave length is given by $\lambda_{\max }=2 \pi \sqrt{3 \epsilon / 2\left|W^{\prime \prime}(0)\right|}$. Fig. 12 shows the dependence of the most unstable wave length and the maximal growth rate on the regularization parameter $\epsilon$.
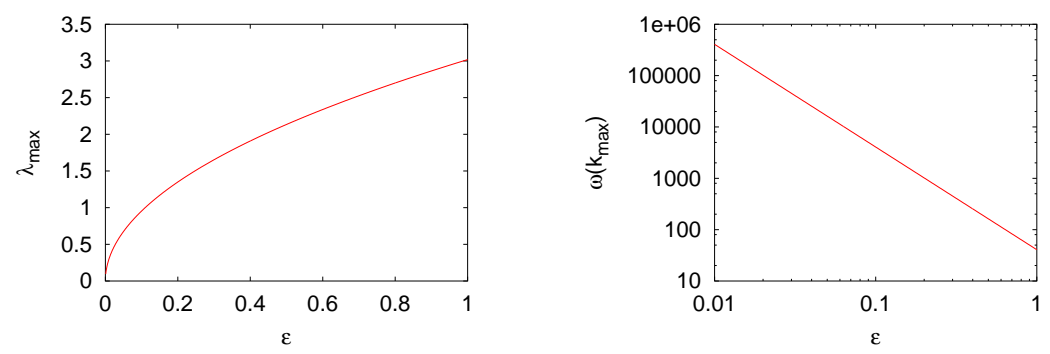

FIG. 12. Left: most unstable wave length depending on the regularization parameter $\epsilon$. Right: maximal growth rate versus $\epsilon$ (log-log plot). In both cases, $\gamma(\theta)=1.0+0.5 \cos (4 \theta)$, which yields $W^{\prime \prime}(0)=-6.5$.

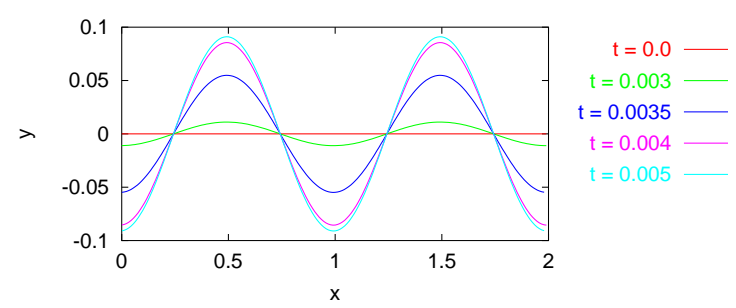

FIG. 13. Time evolution of the surface on a periodic domain of length 2 with $\gamma$ as in 34 and $\epsilon=0.1$ : at the early stage a periodic solution according to the most unstable wave length develops rapidly. The initial surface is a straight line with random perturbation with amplitudes $<10^{-6}$. 


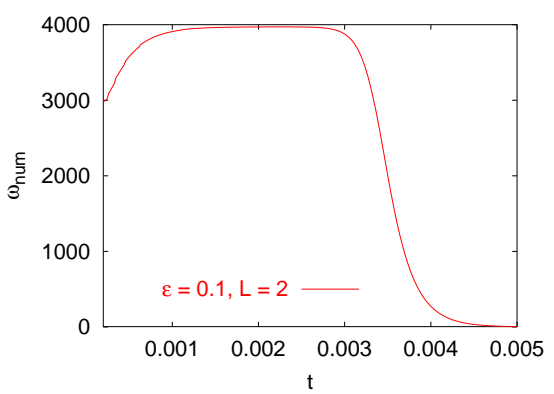

FIG. 14. Numerically computed growth rate over time for the simulation shown in Fig. 13

For a periodic domain of length $L=2$ and a regularization parameter $\epsilon=0.1$, the dominant unstable wave length is $\lambda_{2}=1$. Fig. 13 presents the numerical solution of (7) starting from a randomly perturbed straight line. As theoretically predicted a periodic solution according to the most unstable wave length $\lambda_{2}=1$ develops rapidly. We numerically compute the growth rate from the amplitude $A(t)$ of the numerical solution as

$$
\omega(t)=\frac{1}{\tau}(\ln A(t)-\ln A(t+\tau))
$$

The result is shown in Fig. 14. The maximal growth rate obtained is 3971 and coincides very well with the value $\omega(2 \pi)=3978$ predicted by (44).

\section{Conclusion}

We studied anisotropic surface diffusion with a small corner energy regularization. The introduced regularization rounds corners in the corresponding Wulff shape and sets a new length scale describing the width of the corner. The regularization is the next higher order term in an expansion of the surface free energy density, if $\gamma=\gamma\left(\theta, \partial_{s} \theta\right)$ is assumed to depend not only on the angle $\theta$ but also on its derivative $\partial_{s} \theta$. A connection to a higher order Cahn-Hilliard equation is drawn, which can be derived from a long wave approximation of the full geometric problem.

A discrete scheme for the nonlinear 6th order evolution law is derived for one-dimensional parametric surfaces. The discretization allows the use of linear finite elements.

The convergence of a circle to the Wulff shape of the regularized problem is demonstrated and the evolution of a thermodynamically unstable surface into a hill-valley pattern and its subsequent coarsening is analysed.

In order for the model to be applicable to describe surface modulations of solid films, twodimensional surfaces need to be considered. The theory for the small corner energy regularization in this situation is described in [14]. A numerical algorithm which can deal with parametric surfaces still has to be developed.

Note added in proof. A different parametric discretization of (6) was introduced by M. Siegel, M. J. Miksis and P. W. Voorhees, "Evolution of material voids for highly anisotropic surface energy", J. Mech. Phys. Solids 52 (2004), 13191353. 


\section{Acknowledgments}

We would like to thank Stephen J. Watson for bringing the regularization in [11] to our attention and Brain J. Spencer for explaining the asymptotic analysis in [24]. The work has been supported by BMBF through grant 03VGNKVB.

\section{REFERENCES}

1. B ̈̈NSCh, E., MoRin, P., \& NOChEtTo, R. H. Surface diffusion of graphs: Variational formulation, error analysis and simulation. SIAM J. Numer. Anal. 42 (2004), 773-799. Zbl pre02139899 MR 2084235

2. B̈̈nsch, E., Morin, P., \& Nochetto, R. H. A finite element method for surface diffusion: the parametric case. J. Comput. Phys. 203 (2005), 321-343. Zbl pre02132615 MR 2104399

3. Barrett, J. W., GArCKe, H., \& NÜrnberG, R. Finite element approximations of a phase-field model for surface diffusion of voids in a stressed solid. Tech. rep., Department of Mathematics, Imperial College (2004).

4. BuRger, M. Numerical simulation of anisotropic surface diffusion with curvature-dependent energy. J. Comput. Phys. 203 (2005), 602-625. Zbl pre02167956 MR 2122887

5. Burton, W. K., Cabrera, N., \& Frank, F. C. The growth of crystals and the equilibrium of their surfaces. Philos. Trans. R. Soc. London Ser. A 243 (1951), 299-358. Zbl 0043.23402 MR 0043005

6. CABrera, N. The equilibrium of crystal surfaces. Surf. Sci. 2 (1964), 320.

7. Cahn, J. W., Elliott, C. M., \& Novick-Cohen, A. The Cahn-Hilliard equation with a concentration dependent mobility: motion by minus the Laplacian of the mean curvature. European J. Appl. Math. 7 (1996), 287-301. Zbl 0861.35039 MR 1401172

8. Chopp, D. L. \& Sethian, J. A. Motion by intrinsic Laplacian of curvature. Interfaces Free Bound. 1 (1999), 107-123. Zbl 0938.65144 MR 1865108

9. Clarenz, U., Hausser, F., Rumpf, M., Voigt, A., \& Weikard, U. On level set formulations for anisotropic mean curvature flow and surface diffusion. Multiscale Modeling of Epitaxial Growth, Birkhäuser (2005), 227-238.

10. Deckelnick, K., DZiuk, G., \& Elliott, C. M. Fully discrete semi-implicit second order splitting for anisotropic surface diffusion of graphs. SIAM J. Numer. Anal. (to be published).

11. Di Carlo, A., Gurtin, M. E., \& Podio-Guidugli, P. A regularized equation for anisotropic motionby-curvature. SIAM J. Appl. Math. 52 (1992), 1111. Zbl 0800.73021 MR 1174049

12. DZIUK, G. An algorithm for evolutionary surfaces. Numer. Math. 58 (1991), 603-611. Zbl 0714.65092 MR 1083523

13. EgGleston, J. J. \& VoorheEs, P. W. Ordered growth of nanocrystals via a morphological instability. Appl. Phys. Lett. 80 (2002), 306-308.

14. Gurtin, M. E. \& JabBour, M. E. Interface evolution in three dimensions with curvature-dependent energy and surface diffusion: Interface-controlled evolution, phase transition, epitaxial growth of elastic films. Arch. Rat. Mech. Anal. 163 (2002), 171-208. Zbl 1053.74005 MR 1912105

15. Hausser, F. \& Voigt, A. Weakly anisotropic surface diffusion, a numerical approach by parametric finite elements. Tech. rep., SFB 611, Univ. Bonn (2003).

16. Hausser, F. \& Voigt, A. A numerical scheme for regularized anisotropic curve shortening flow. Appl. Math. Lett. (2004), to appear.

17. Herring, C. Some theorems on the free energies of crystal surfaces. Phys. Rev. 82 (1951), 87-93. Zbl 0042.23201

18. Herring, C. Structure and Properties of Solid Surfaces. Univ. of Chicago Press (1963), 5-81.

19. LiU, F. \& Metiu, H. Dynamics of phase separation of crystal surfaces. Phys. Rev. B 48 (1993), 58085817. 
20. RÄtz, A., Ribalta, A., \& Voigt, A. Surface evolution of elastically stressed films under deposition by a diffuse interface model. J. Comput. Phys. (to be published).

21. Savina, T. V., Golovin, A. A., Davis, S. H., Nepomnyashchy, A. A., And Voorhees, P. W. Faceting of a growing crystal surface by surface diffusion. Phys. Rev. E 67 (2003), 021606.

22. Schmidt, A. \& Siebert, K. G. ALBERT—software for scientific computations and applications. Acta Math. Univ. Comen. 70 (2001), 105-122. Zbl 0993.65134 MR 1865363

23. SmereKa, P. Semi-implicit level-set methods for curvature and surface diffusion motion. J. Sci. Comput. 19 (2003), 439-455. Zbl 1035.65098 MR 2028853

24. Spencer, B. J. Asymptotic solutions for the equilibrium crystal shape with small corner energy regularization. Phys. Rev. E 69 (2004), 011603.

25. Steward, J. \& Goldenfeld, N. Spinodal decomposition of a crystal surface. Phys. Rev. A 46 (1992), $6505-6511$.

26. WAtson, S. J. Crystal growth, coarsening and the convective Cahn-Hilliard equation. P. Colli et al. (eds.), Free Boundary Problems (Trento, 2002), Birkhäuser (2004), 329-341. Zbl 1040.35078 MR 2044584

27. WulfF, G. Zur Frage der Geschwindigkeit des Wachstums und der Auflösung der Kristallflächen. Z. F. Kristallog. 34 (1901), 449-530. 